\title{
The chromomagnetic operator on the lattice
}

\author{
M. Constantinou ${ }^{a}$, M. Costa ${ }^{a}$, R. Frezzotti $^{b}$, V. Lubicz ${ }^{c}$, G. Martinelli ${ }^{d}$, D. Meloni ${ }^{c}$, \\ H. Panagopoulos ${ }^{* a}$, S. Simula ${ }^{c}$
}

${ }^{a}$ Department of Physics, University of Cyprus, CY-1678 Nicosia, Cyprus

${ }^{b}$ Dipartimento di Fisica, Università di Roma "Tor Vergata" and INFN Sezione "Tor Vergata", I-00133 Rome, Italy

${ }^{c}$ Dipartimento di Fisica, Università Roma Tre, and INFN, Sezione di Roma Tre, I-00146 Rome, Italy

${ }^{d}$ SISSA, I-34136 Trieste, Italy

E-mail: constantinou.martha@ucy.ac.cy, kosta.marioseucy.ac.cy

roberto.frezzottieroma2.infn.it lubiczefis.uniroma3.it,

guido.martinellidsissa.it meloniefis.uniroma3.it, hariseucy.ac.cy,

simulaeroma3.infn.it

We study matrix elements of the "chromomagnetic" operator on the lattice. This operator is contained in the strangeness-changing effective Hamiltonian which describes electroweak effects in the Standard Model and beyond.

Having dimension 5, the chromomagnetic operator is characterized by a rich pattern of mixing with other operators of equal and lower dimensionality, including also non gauge invariant quantities; it is thus quite a challenge to extract from lattice simulations a clear signal for the hadronic matrix elements of this operator.

We compute all relevant mixing coefficients to one loop in lattice perturbation theory; this necessitates calculating both 2-point (quark-antiquark) and 3-point (gluon-quark-antiquark) Green's functions at nonzero quark masses. We use the twisted mass lattice formulation, with Symanzik improved gluon action.

For a comprehensive presentation of our results, along with detailed explanations and a more complete list of references, we refer to our forthcoming publication [1].

31st International Symposium on Lattice Field Theory LATTICE 2013

July 29 - August 3, 2013

Mainz, Germany

\footnotetext{
*Speaker.
} 


\section{Introduction}

The electroweak effective Hamiltonian describing stangeness changing $(\Delta S=1)$ processes, in the Standard Model (SM) and beyond, contains four "magnetic" operators of dimension 5:

$$
\begin{gathered}
H_{\mathrm{eff}}^{\Delta S=1, d=5}=\sum_{i= \pm}\left(C_{\gamma}^{i} Q_{\gamma}^{i}+C_{g}^{i} Q_{g}^{i}\right)+\text { h.c. } \\
Q_{\gamma}^{ \pm}=\frac{Q_{d} e}{16 \pi^{2}}\left(\bar{s}_{L} \sigma^{\mu v} F_{\mu \nu} d_{R} \pm \bar{s}_{R} \sigma^{\mu v} F_{\mu \nu} d_{L}\right), \quad Q_{g}^{ \pm}=\frac{g}{16 \pi^{2}}\left(\bar{s}_{L} \sigma^{\mu v} G_{\mu \nu} d_{R} \pm \bar{s}_{R} \sigma^{\mu v} G_{\mu \nu} d_{L}\right)
\end{gathered}
$$

The coefficients $C_{\gamma}^{i}$ and $C_{g}^{i}$, multiplying the electromagnetic (EMO) and chromomagnetic (CMO) operators, respectively, may be calculated perturbatively via the OPE; they are suppressed within the SM, but become more pronounced beyond the SM, e.g. through penguin diagrams in SUSY.

The matrix elements of the $\mathrm{CMO}$ are parameterized as [2]:

$$
\begin{aligned}
\left\langle\pi^{0}\left|Q_{g}^{+}\right| K^{0}\right\rangle & =\frac{-11}{32 \sqrt{2} \pi^{2}} \frac{M_{K}^{2}\left(p_{\pi} \cdot p_{K}\right)}{m_{s}+m_{d}} B_{g 1} \\
\left\langle\pi^{+} \pi^{-}\left|Q_{g}^{-}\right| K^{0}\right\rangle & =\frac{11 \mathrm{i}}{32 \pi^{2}} \frac{M_{K}^{2} M_{\pi}^{2}}{f_{\pi}\left(m_{s}+m_{d}\right)} B_{g 2} \\
\left\langle\pi^{+} \pi^{+} \pi^{-}\left|Q_{g}^{+}\right| K^{+}\right\rangle & =\frac{-11}{16 \pi^{2}} \frac{M_{K}^{2} M_{\pi}^{2}}{f_{\pi}^{2}\left(m_{s}+m_{d}\right)} B_{g 3}
\end{aligned}
$$

These matrix elements are relevant for the study of $K^{0}-\bar{K}^{0}$ mixing, $\varepsilon^{\prime} / \varepsilon$, the $\Delta I=1 / 2$ rule, and $K \rightarrow 3 \pi$ decays. To leading order in $\chi \mathrm{PT}$, the $B$-parameters are all related [B]:

$$
Q_{g}^{ \pm}=\frac{11}{256 \pi^{2}} \frac{f_{\pi}^{2} M_{K}^{2}}{m_{s}+m_{d}} B_{g}\left[U\left(D_{\mu} U^{\dagger}\right)\left(D^{\mu} U\right) \pm\left(D_{\mu} U^{\dagger}\right)\left(D^{\mu} U\right) U^{\dagger}\right]_{23}
$$

Thus, a lattice study of, say, Eq. (1.2), provides information for Eqs. (1.3), 1.4) as well.

The EMO has been studied in simulations with $N_{f}=0$ [4] and $N_{f}=2$ [5] dynamical flavors, focusing on:

$$
\left\langle\pi^{0}\left|Q_{\gamma}^{+}\right| K^{0}\right\rangle=\mathrm{i} \frac{Q_{d} e \sqrt{2}}{16 \pi^{2} M_{K}} p_{\pi}^{\mu} p_{K}^{v} F_{\mu \nu} B_{T} R_{T}\left(q^{2}\right) \quad\left[R_{T}(0)=1\right]
$$

The parameter $B_{T}$ appears, e.g., in the branching ratio of $K_{L} \rightarrow \pi^{0} e^{+} e^{-}$in SUSY models.

\section{Operator Mixing - Lattice Action - Symmetries}

A formidable issue in the study of the CMO is the fact that it mixes with a large number of other operators under renormalization. Even in dimensional regularization (DR), which has the simplest mixing pattern, the $\operatorname{CMO}\left(\mathscr{O}_{C M} \equiv \mathscr{O}_{1}\right)$ mixes with a total of 9 other operators $\left(\mathscr{O}_{2}-\mathscr{O}_{10}\right)$, forming a basis of dimension-five, Lorentz scalar operators with the same flavor content as the CMO. Among them, there are also gauge noninvariant operators $\left(\mathscr{O}_{9}, \mathscr{O}_{10}\right)$; these are BRST invariant and vanish by the equations of motion, as required by renormalization theory.

$$
\begin{aligned}
& \mathscr{O}_{1}=g \bar{\psi}_{s} \sigma_{\mu \nu} G_{\mu \nu} \psi_{d} \\
& \mathscr{O}_{6}=\bar{\psi}_{s}\left(\vec{D}+m_{d}\right)^{2} \psi_{d}+\bar{\psi}_{s}\left(-\overleftarrow{\not D}+m_{s}\right)^{2} \psi_{d} \\
& \mathscr{O}_{2}=\left(m_{d}^{2}+m_{s}^{2}\right) \bar{\psi}_{s} \psi_{d} \\
& \mathscr{O}_{7}=m_{s} \bar{\psi}_{s}\left(\vec{D}+m_{d}\right) \psi_{d}+m_{d} \bar{\psi}_{s}\left(-\overleftarrow{D}+m_{s}\right) \psi_{d} \\
& \mathscr{O}_{3}=m_{d} m_{s} \bar{\psi}_{s} \psi_{d} \\
& \mathscr{O}_{8}=m_{d} \bar{\psi}_{s}\left(\vec{D}+m_{d}\right) \psi_{d}+m_{s} \bar{\psi}_{s}\left(-\overleftarrow{D}+m_{s}\right) \psi_{d} \\
& \mathscr{O}_{4}=\bar{\psi}_{s} \overleftarrow{D}_{\mu} \vec{D}_{\mu} \psi_{d} \\
& \mathscr{O}_{9}=\bar{\psi}_{s} \overleftarrow{\partial}\left(\vec{D}+m_{d}\right) \psi_{d}-\bar{\psi}_{s}\left(-\overleftarrow{D}+m_{s}\right) \vec{\partial} \psi_{d} \\
& \mathscr{O}_{5}=\bar{\psi}_{s}\left(-\overleftarrow{D}+m_{s}\right)\left(\not \vec{D}+m_{d}\right) \psi_{d} \\
& \mathscr{O}_{10}=\bar{\psi}_{s} \overrightarrow{\not \partial}\left(\vec{D}+m_{d}\right) \psi_{d}-\bar{\psi}_{s}\left(-\overleftarrow{D}+m_{s}\right) \overleftarrow{\not \partial} \psi_{d}
\end{aligned}
$$


On the lattice, the mixing pattern can become considerably more complicated, given that certain symmetries are violated; there can be mixing with additional operators of dimension five (with finite coefficients) or less (with power-divergent coefficients). A generic hypercubic- and gaugeinvariant lattice discretization will result in mixing with $2+8+32$ candidate operators of dimension $3,4,5$, respectively. It is thus imperative to make a judicious choice of lattice action, with a large set of discrete symmetries, so as to exclude as many as possible of these candidates.

We have adopted the twisted mass action for valence quarks and the Osterwalder - Seiler action for sea quarks [6] (along with a compensating ghost action for valence quarks). For our one-loop perturbative calculation we only need the valence quark action, which reads (in the physical basis):

$$
\begin{gathered}
S_{F}\left[\psi_{f}, \bar{\psi}_{f}, U\right]=a^{4} \sum_{f} \sum_{x} \bar{\psi}_{f}(x)\left[\gamma \cdot \widetilde{\nabla}-i \gamma_{5} W_{\mathrm{cr}}\left(r_{f}\right)+m_{f}\right] \psi_{f}(x) \\
\gamma \cdot \widetilde{\nabla} \equiv \frac{1}{2} \sum_{\mu} \gamma_{\mu}\left(\nabla_{\mu}^{\star}+\nabla_{\mu}\right) \quad W_{\mathrm{cr}}\left(r_{f}\right) \equiv-a \frac{r_{f}}{2} \sum_{\mu} \nabla_{\mu}^{\star} \nabla_{\mu}+M_{\mathrm{cr}}\left(r_{f}\right)
\end{gathered}
$$

( $r_{f}$ : Wilson parameter for flavour $f=u, d, s ; M_{\mathrm{cr}}\left(r_{f}\right)=-M_{\mathrm{cr}}\left(-r_{f}\right)$ : corresponding critical mass).

For gluons we have used the Symanzik improved action; for our perturbative results we employed several standard choices of values for the Symanzik coefficients appearing in that action [1].

A number of discrete symmetries [6] are present in our action; the CMO is invariant - up to a possible minus sign - under them, and the same must then hold for all other operators which mix with the CMO. As a result, alongside the 10 operators which mix in DR, only 3 additional ones appear on the lattice, and they all have dimension less than five:

$$
\mathscr{O}_{11}=i r_{d} \bar{\psi}_{s} \gamma_{5}\left(\not \vec{D}+m_{d}\right) \psi_{d}+i r_{s} \bar{\psi}_{s}\left(-\overleftarrow{D}+m_{s}\right) \gamma_{5} \psi_{d}, \mathscr{O}_{12}=i\left(r_{d} m_{d}+r_{s} m_{s}\right) \bar{\psi}_{s} \gamma_{5} \psi_{d}, \mathscr{O}_{13}=\bar{\psi}_{s} \psi_{d}
$$

\section{Renormalization Matrix}

Renormalized operators $\mathscr{O}_{i}^{R}$ are related to bare ones $\mathscr{O}_{i}$ via a $13 \times 13$ renormalization matrix $Z$ :

$$
\mathscr{O}_{i}=\sum_{j=1}^{13} Z_{i j} \mathscr{O}_{j}^{R} \quad\left(\mathscr{O}=Z \mathscr{O}^{R}, \mathscr{O}^{R}=Z^{-1} \mathscr{O}\right)
$$

The matrix elements $Z_{i j}$ depend both on the regularization $X$ ( $X=L$ (lattice), $D R$ (dimensional), etc.) and on the renormalization scheme $Y\left(Y=\overline{\mathrm{MS}}, R I^{\prime}\right.$, etc.); where confusion might arise, one should denote them as $Z_{i j}^{X, Y}$. At tree level: $Z=\mathbb{1}$. For $\mathscr{O}_{1}^{R}$, we only need the first row of $Z^{-1}$ (and thus, to one loop, only the first row or $Z$ : $\left.Z_{1 i}\right)$. Clearly: $Z_{1,1}=1+\mathscr{O}\left(g^{2}\right), Z_{1 i}=\mathscr{O}\left(g^{2}\right)(i>1)$.

To obtain $Z_{1 i}$, we have calculated, to one loop and in an arbitrary covariant gauge, the 2-point (quark-antiquark) and 3-point (quark-antiquark-gluon) bare amputated Green's functions of $\mathscr{O}_{1}$; these are related to the corresponding renormalized Green's functions through:

$$
\begin{array}{lc}
\left\langle\psi^{R} \mathscr{O}_{1}^{R} \bar{\psi}^{R}\right\rangle_{\mathrm{amp}}=Z_{\psi} \sum_{i=1}^{13}\left(Z^{-1}\right)_{1 i}\left\langle\psi \mathscr{O}_{i} \bar{\psi}\right\rangle_{\mathrm{amp}}, & \psi=Z_{\psi}^{1 / 2} \psi^{R} \\
\left\langle\psi^{R} \mathscr{O}_{1}^{R} \bar{\psi}^{R} A_{v}^{R}\right\rangle_{\mathrm{amp}}=Z_{\psi} Z_{A}^{1 / 2} \sum_{i=1}^{13}\left(Z^{-1}\right)_{1 i}\left\langle\psi \mathscr{O}_{i} \bar{\psi} A_{v}\right\rangle_{\mathrm{amp}}, & A_{v}=Z_{A}^{1 / 2} A_{v}^{R}
\end{array}
$$

The renormalization functions $Z_{\psi}, Z_{A}$ (as well as those for the coupling constant $\left(Z_{g}\right)$, the fermion mass $\left(Z_{m}\right)$, and the ghost field $\left(Z_{c}\right)$ ) were not all available for the actions considered in 
this work, and had to be calculated as a prerequisite. We mention in passing that $Z_{\psi}$ and $Z_{m}$ do not depend on flavor in mass-independent schemes. We also note that both the 2-point and 3-point functions are necessary in order to fix all $Z_{1 i}$, but they are also sufficient.

[An alternative definition of the CMO: $\widetilde{\mathscr{O}}_{C M} \equiv m \mathscr{O}_{C M}$ appears in the study of 4-fermi operators. In this case, the renormalization matrix reads: $\tilde{Z}_{i j}=Z_{m} Z_{i j}\left(m^{R} \equiv Z_{m}^{-1} m\right)$. Similarly, a factor of $Z_{g}$ must be included in $Z_{i j}$, if Green's functions are computed using: $(1 / g) \mathscr{O}_{C M}$, rather: $\mathscr{O}_{C M}$.]

The one-particle irreducible (1PI) Feynman diagrams contributing to the 2-point and 3-point Green's functions of $\mathscr{O}_{1}$ are shown in the left and right panels of Figure 1, respectively.
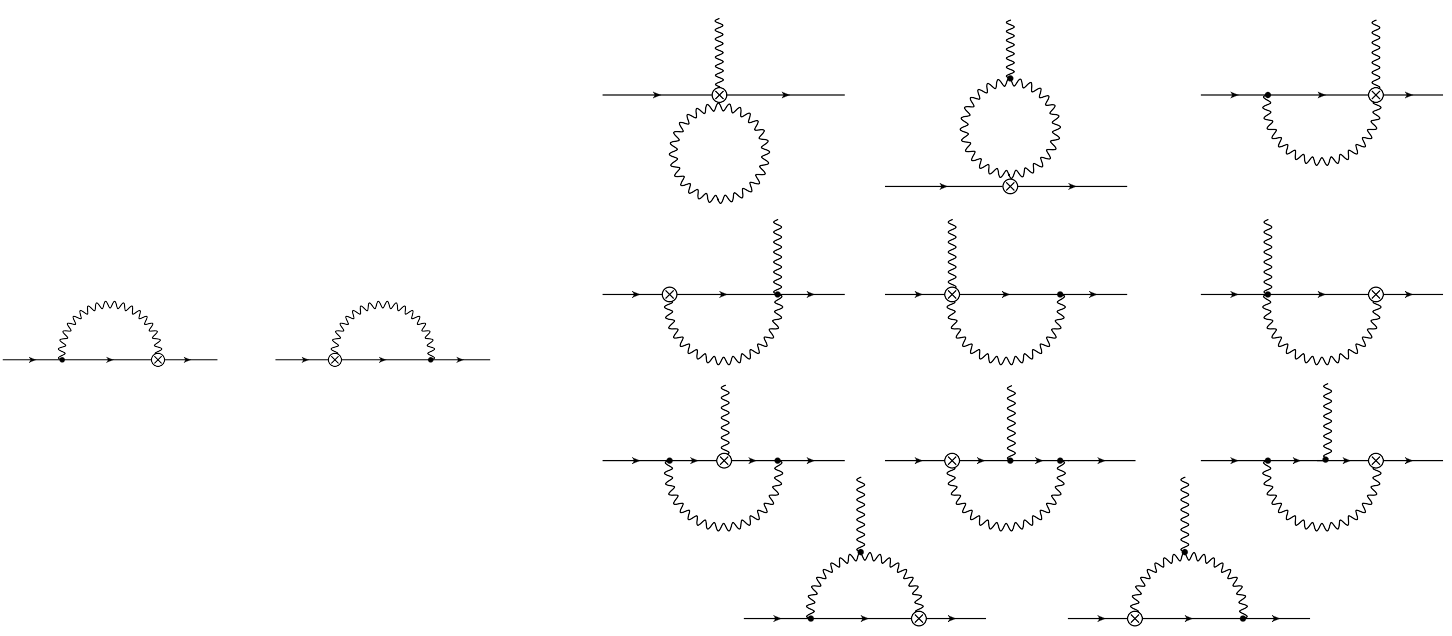

Figure 1: 1PI one-loop diagrams contributing to the 2-point and 3-point Green's functions. A wavy (solid) line represents gluons (quarks); an operator insertion is denoted by $\otimes$.

\section{Results}

We calculated the 2- and 3-point bare Green's functions of Eq. (3.2), first in DR and then in the far more complicated case of the lattice. The purpose of the calculation in DR is twofold: First, it provides the mixing coefficients $Z_{1 i}^{D R, \overline{\mathrm{MS}}}$, which are interesting on their own right; second, and most important, it leads to the renormalized Green's functions in $\overline{\mathrm{MS}}$, which are then necessary for extracting the real quantities of interest: $Z_{1 i}^{L, \overline{\mathrm{MS}}}$.

\subsection{Dimensional Regularization and $\overline{\mathrm{MS}}$ Renormalization}

In $D=4-2 \varepsilon$ dimensions, renormalizability requires that the $\mathscr{O}(1 / \varepsilon)$, 1PI part in the bare Green's functions of Eq. (3.2) has polynomial dependence on $m_{s}, m_{d}, q_{s}, q_{d}, q_{A}\left(q_{s} / q_{d} / q_{A}\right.$ : momenta of the external antiquark/quark/gluon). In fact, there appear in total $7+4$ types of such dependence, as follows:

$$
\begin{aligned}
\left.\left\langle\psi \mathscr{O}_{1} \bar{\psi}\right\rangle_{\text {amp }}^{1-\text { loop }}\right|_{1 / \varepsilon}= & \rho_{1}\left(q_{s}^{2}+q_{d}^{2}\right)+\rho_{2}\left(m_{s}^{2}+m_{d}^{2}\right)+\rho_{3} i\left(m_{d} q_{d}+m_{s} q_{s}\right) \\
& +\rho_{4} i\left(m_{s} q_{d}+m_{d} q_{s}\right)+\rho_{5} q_{s} \cdot q_{d}+\rho_{6} q_{s} q_{d}+\rho_{7} m_{s} m_{d} \\
\left.\left\langle\psi \mathscr{O}_{1} \bar{\psi} A_{v}\right\rangle_{\text {amp }}^{1-l o o p}\right|_{1 / \varepsilon}= & R_{1} g\left(q_{s}+q_{d}\right)_{v}+R_{2} g\left(\gamma_{v} q_{d}+q_{s} \gamma_{v}\right) \\
& +R_{3} i g\left(m_{s}+m_{d}\right) \gamma_{v}+R_{4}\left(-2 i g \sigma_{\rho v} q_{A \rho}\right)
\end{aligned}
$$


There exist also 1-particle reducible diagrams contributing to the 3-point function, both at tree level and at one loop; these contain non-polynomial $\mathscr{O}(1 / \varepsilon)$ terms, which however cancel by virtue of the 2-point relation. Computing the coefficients $\rho_{1}-\rho_{7}, R_{1}-R_{4}$ we find:

$$
\begin{gathered}
\rho_{1}=\frac{g^{2} C_{F}}{16 \pi^{2}} \frac{1}{\varepsilon}(-3) \quad \rho_{2}=\frac{g^{2} C_{F}}{16 \pi^{2}} \frac{1}{\varepsilon}(-6) \quad \rho_{3}=\frac{g^{2} C_{F}}{16 \pi^{2}} \frac{1}{\varepsilon}(3) \quad \rho_{4}=\rho_{5}=\rho_{6}=\rho_{7}=0 \\
\left\{R_{1}, R_{2}, R_{3}, R_{4}\right\}=\frac{g^{2}}{16 \pi^{2}} \frac{1}{\varepsilon}\left\{-6 C_{F}, \frac{3 N_{c}}{4},\left(\frac{-3}{2 N_{c}}+\frac{3 N_{c}}{4}\right),\left(\frac{1}{N_{c}}-\frac{\alpha}{2 N_{c}}+\frac{7 N_{c}}{4}+\frac{3 \alpha N_{c}}{4}\right)\right\}
\end{gathered}
$$

$\left(N_{c}\right.$ : number of colors, $C_{F}=\left(N_{c}^{2}-1\right) /\left(2 N_{c}\right), \alpha$ : gauge parameter).

Demanding that all $\mathscr{O}(1 / \varepsilon)$ dependence on the right-hand sides of Eqs. (3.2) disappears (as it ought to, since the corresponding renormalized Green's functions on the left-hand sides must be finite) provides $7+4$ constraint equations on the 10 coefficients $Z_{1 i}$. This set of equations is self-consistent and complete; solving them, we obtain:

$$
\begin{array}{ll}
Z_{1,1}^{D R, \overline{\mathrm{MS}}}=1+\frac{g^{2}}{16 \pi^{2}} \frac{1}{\varepsilon}\left(-\frac{N_{c}}{2}+\frac{5}{2 N_{c}}\right) & Z_{1,2}^{D R, \overline{\mathrm{MS}}}=-2 Z_{1,10}^{D R, \overline{\mathrm{MS}}}=\frac{g^{2}}{16 \pi^{2}} \frac{1}{\varepsilon}\left(-3 N_{c}+\frac{3}{N_{c}}\right) \\
Z_{1,5}^{D R, \overline{\mathrm{MS}}}=\frac{g^{2}}{16 \pi^{2}} \frac{1}{\varepsilon}\left(\frac{2 N_{c}}{3}-\frac{3}{N_{c}}\right) & Z_{1,7}^{D R, \overline{\mathrm{MS}}}=-Z_{1,9}^{D R, \overline{\mathrm{MS}}}=\frac{g^{2}}{16 \pi^{2}} \frac{1}{\varepsilon}\left(-\frac{3 N_{c}}{4}+\frac{3}{2 N_{c}}\right) \\
Z_{1,3}^{D R, \overline{\mathrm{MS}}}=Z_{1,4}^{D R, \overline{\mathrm{MS}}}=Z_{1,6}^{D R, \overline{\mathrm{MS}}}=Z_{1,8}^{D R, \overline{\mathrm{MS}}}=0
\end{array}
$$

An immediate, well-known by-product of $Z_{1,1}^{D R, \overline{\mathrm{MS}}}$ is the anomalous dimension $\widetilde{\gamma}_{C M}$ for the operator $\widetilde{\mathscr{O}}_{C M}: \widetilde{\gamma}_{C M}=g^{2} /\left(16 \pi^{2}\right) \cdot\left(4 N_{c}-8 / N_{c}\right)$. We note that the mixing coefficients for the gauge noninvariant operators $\mathscr{O}_{9}, \mathscr{O}_{10}$ do not vanish.

The $\mathscr{O}\left(\varepsilon^{0}\right)$ parts of the right-hand side of Eqs. (3.2) are the $\overline{\mathrm{MS}}$-renormalized Green's functions; while they were not necessary for $Z_{1 i}^{D R, \overline{\mathrm{MS}}}$, we do need them for $Z_{1 i}^{L, \overline{\mathrm{MS}}}$ below.

\subsection{Lattice Regularization and $\overline{\mathrm{MS}}$ Renormalization}

The relations which we must turn to now are formally the same as the ones we studied in the previous subsection (Eqs. (3.2)); however all renormalization functions $Z$ now stand for $Z^{L, \overline{\mathrm{MS}}}$, and the bare Green's functions on the right-hand sides must be calculated using the lattice regularization. The $\overline{\mathrm{MS}}$-renormalized Green's functions on the left-hand sides coincide with those which we calculated in the previous subsection, since they must be regularization-independent.

Renormalizability implies that, modulo terms which vanish as $a \rightarrow 0,\left\langle\psi^{R} \mathscr{O}_{1}^{R} \bar{\psi}^{R}\right\rangle_{\text {amp }}-\left\langle\psi \mathscr{O}_{1} \bar{\psi}\right\rangle_{\text {amp }}$ is polynomial in $m$ 's, $q$ 's (of $2^{\text {nd }}$ degree, but also $a^{-1} \cdot\left(1^{\text {st }}\right), a^{-2} \cdot\left(0^{\text {th }}\right)$ ):

$$
\begin{gathered}
\left\langle\psi^{R} \mathscr{O}_{1}^{R} \bar{\psi}^{R}\right\rangle_{\mathrm{amp}}-\left\langle\psi \mathscr{O}_{1} \bar{\psi}\right\rangle_{\mathrm{amp}}=\rho_{1}\left(q_{s}^{2}+q_{d}^{2}\right)+\rho_{2}\left(m_{s}^{2}+m_{d}^{2}\right)+\rho_{3} i\left(m_{d} q_{d}+m_{s} q_{s}\right)+\rho_{4} i\left(m_{s} q_{d}+m_{d} q_{s}\right) \\
\quad+\rho_{5} q_{s} \cdot q_{d}+\rho_{6} q_{s} q_{d}+\rho_{7} m_{s} m_{d}+\rho_{\mathbf{8}}\left(r_{d} \gamma_{5} q_{d}+r_{s} q_{s} \gamma_{5}\right)+\rho_{9} i\left(r_{d} m_{d}+r_{s} m_{s}\right) \gamma_{5}+\rho_{10} \cdot 1
\end{gathered}
$$

Similarly, $\left\langle\psi^{R} \mathscr{O}_{1}^{R} \bar{\psi}^{R} A_{v}^{R}\right\rangle_{\text {amp }}-\left\langle\psi \mathscr{O}_{1} \bar{\psi} A_{v}\right\rangle_{\text {amp }}$ must be polynomial $\left(1^{\text {st }}\right.$ degree, but also $\left.a^{-1} \cdot\left(0^{\text {th }}\right)\right)$ :

$$
\begin{aligned}
\left\langle\psi^{R} \mathscr{O}_{1}^{R} \bar{\psi}^{R} A_{v}^{R}\right\rangle_{\mathrm{amp}}-\left\langle\psi \mathscr{O}_{1} \bar{\psi} A_{v}\right\rangle_{\mathrm{amp}}= & R_{1} g\left(q_{s}+q_{d}\right)_{v}+R_{2} g\left(\gamma_{v} q_{d}+q_{s} \gamma_{v}\right)+R_{3} i g\left(m_{s}+m_{d}\right) \gamma_{v} \\
& +R_{4}\left(-2 i g \sigma_{\rho v} q_{A \rho}\right)+\mathbf{R}_{5} g\left(r_{d}-r_{s}\right) \gamma_{5} \gamma_{v}
\end{aligned}
$$


The $10+5$ coefficients $\rho_{i}, R_{i}$ depend on $a$ as: $a^{-2}, a^{-1}, \log (a \bar{\mu})$ (the scale $\bar{\mu}$ appears through the $\overline{\mathrm{MS}}$-renormalized Green's functions); they also depend on: $N_{c}, \alpha$, and the Symanzik coefficients.

Thus, enforcing Eqs. (3.2) leads to $10+5$ constraints for the 13 functions $Z_{1 i}$, in such a way as to absorb the above polynomial differences. These constraints are self-consistent and complete.

It is a highly nontrivial task to show that the left-hand sides of Eqs. (4.5, 4.6) are polynomial. This is especially true for Eq. (4.6): The $\overline{\mathrm{MS}}$-renormalized 3-point function has already an extremely complicated dependence on momenta and masses (involving Spence functions even for $m=0$ ), while the lattice bare 3-point function contains $\sim 10^{5}$ loop integrals depending on masses and external momenta. One relatively easy way to show the above property is to make a special nondegenerate choice for the external momenta, e.g. the "democratic" one: $q_{s}-q_{d}+q_{A}=0, q_{s}^{2}=q_{d}^{2}=q_{A}^{2}=\bar{\mu}^{2}$. In order to subject our results to as stringent a test as possible, we showed polynomiality without making any simplifying assumptions on the values of the external momentum 4-vectors, not even momentum conservation. An independent (far simpler) check on the lattice Green's functions is that the coefficients of $\log (a)$ must match those of $-1 /(2 \varepsilon)$ in DR.

Solving the constraint equations we find, in the case of the tree-level Symanzik gluon action:

$$
\begin{aligned}
& Z_{1,1}^{L, \overline{\mathrm{MS}}}=1+\frac{g^{2}}{16 \pi^{2}}\left(N_{c}\left(-12.8455+\frac{1}{2} \log \left(a^{2} \bar{\mu}^{2}\right)\right)+\frac{1}{N_{c}}\left(9.3779-\frac{5}{2} \log \left(a^{2} \bar{\mu}^{2}\right)\right),\right. \\
& Z_{1,2}^{L, \overline{\mathrm{MS}}}=\frac{g^{2} C_{F}}{16 \pi^{2}}\left(2.7677+6 \log \left(a^{2} \bar{\mu}^{2}\right)\right), Z_{1,3}^{L, \overline{\mathrm{MS}}}=0, Z_{1,4}^{L, \overline{\mathrm{MS}}}=0, \\
& Z_{1,5}^{L, \overline{\mathrm{MS}}}=\frac{g^{2}}{16 \pi^{2}}\left(N_{c}\left(5.3894-\frac{3}{2} \log \left(a^{2} \bar{\mu}^{2}\right)\right)+\frac{1}{N_{c}}\left(-5.5061+3 \log \left(a^{2} \bar{\mu}^{2}\right)\right)\right), Z_{1,6}^{L, \overline{\mathrm{MS}}}=0, \\
& Z_{1,7}^{L, \overline{\mathrm{MS}}}=-Z_{1,9}^{L, \overline{\mathrm{MS}}}=-\frac{Z_{5}^{L, \overline{\mathrm{MS}}}}{2}, Z_{1,8}^{L, \overline{\mathrm{MS}}}=\frac{g^{2} C_{F}}{16 \pi^{2}}(-3.9654), Z_{1,10}^{L, \overline{\mathrm{MS}}}=\frac{g^{2} C_{F}}{16 \pi^{2}}\left(5.5061-3 \log \left(a^{2} \bar{\mu}^{2}\right)\right), \\
& Z_{1,11}^{L, \overline{\mathrm{MS}}}=\frac{1}{a} \frac{g^{2} C_{F}}{16 \pi^{2}}(-4.0309)=-Z_{1,12}^{L, \overline{\mathrm{MS}}}, Z_{1,13}^{L, \overline{\mathrm{MS}}}=\frac{1}{a^{2}} \frac{g^{2} C_{F}}{16 \pi^{2}}(47.7929)
\end{aligned}
$$

Systematic errors coming from numerical loop integration are much smaller than the precision presented in the above results. Also, certain mixing coefficients vanish at one loop, but not beyond.

\subsection{Non-perturbative results - Preliminary}

In the calculation of on-shell matrix elements, by virtue of the equations of motion, some of the operators $\mathscr{O}_{1}-\mathscr{O}_{13}$ will not appear. The remaining ones: $\mathscr{O}_{1}, \mathscr{O}_{2}, \mathscr{O}_{3}, \mathscr{O}_{4}, \mathscr{O}_{12}, \mathscr{O}_{13}$ will be present, and it is imperative to have a stringent estimate of the corresponding mixing coefficients. For operators of the same dimensionality as the chromomagnetic one, i.e. $\mathscr{O}_{1}, \mathscr{O}_{2}, \mathscr{O}_{3}, \mathscr{O}_{4}$, our one-loop results are expected to provide satisfactory accuracy; however, for operators of lower dimensionality $\left(\mathscr{O}_{12}, \mathscr{O}_{13}\right)$, given that their coefficients are power divergent, perturbation theory is expected to provide only a ballpark estimate at best. Fortunately, it is precisely for the coefficients of these latter operators that we can have best access to non-perturbative estimates.

Imposing conditions such as:

$$
\begin{gathered}
\lim _{m_{s}, m_{d} \rightarrow 0}\left\langle\pi(0)\left|\mathscr{O}_{1}^{\mathrm{sub}}\right| K(0)\right\rangle=\lim _{m_{s}, m_{d} \rightarrow 0}\left\langle\pi(0)\left|\mathscr{O}_{1}+\frac{c_{13}}{a^{2}} \mathscr{O}_{13}\right| K(0)\right\rangle=0 \\
\left\langle 0\left|\mathscr{O}_{1}^{\mathrm{sub}}\right| K(0)\right\rangle_{m_{s}, m_{d}}=\left\langle 0\left|\mathscr{O}_{1}+\frac{c_{13}}{a^{2}} \mathscr{O}_{13}+\frac{c_{12}}{a} \mathscr{O}_{12}\right| K(0)\right\rangle_{m_{s}, m_{d}}=0
\end{gathered}
$$


we can fit the values of $c_{13}\left(g_{0}\right), c_{12}\left(g_{0}\right)$ to data from simulations with varying quark masses.

In a preliminary series of simulations [1], we have extracted $c_{13}$ at different values of the coupling $\left(\beta \equiv 6 / g_{0}^{2}=1.90,1.95,2.10\right.$ ). The results for $c_{13}$ closely follow a quadratic dependence on $g_{0}$, thus resembling a one-loop effect; nevertheless there is a notable difference, as was expected:

$$
Z_{1,13}^{\text {non-pert }} \sim a^{-2} \frac{g^{2} C_{F}}{16 \pi^{2}}(33.7) \quad Z_{1,13}^{\text {pert }}=a^{-2} \frac{g^{2} C_{F}}{16 \pi^{2}}(47.793)
$$

[For a discussion on the possible choices for the coupling constant, see Ref. [1].]

\section{Checks - Extensions}

Besides a series of controls which we have applied to our results, some further ones may be applied: (i) A calculation of 4-point Green's functions will provide important consistency checks, but no new information, on $Z_{1 i}$. On the other hand, 5-point functions and beyond are irrelevant: Being superficially convergent, they have a straightforward continuum limit. (ii) Non-perturbative estimates of all mixing coefficients would be very important cross checks.

Depending on the method one wishes to employ for computing matrix elements of the CMO non-perturbatively, a renormalization scheme other than $\overline{\mathrm{MS}}$ may be more appropriate. In particular, one may employ an extension of the RI' scheme, in which RI'-like conditions need to be imposed on both 2-point and 3-point functions. The new mixing coefficients $Z_{i j}^{L, R I^{\prime}}$ are related to $Z_{i j}^{L, \overline{\mathrm{MS}}}$ via a $(13 \times 13)$ regularization-independent conversion matrix, whose elements are finite functions of the renormalized coupling. In fact, all relevant matrix elements are directly obtainable from our results on the renormalized Green's functions, with no further calculation required.

A further extension of the present work would be to apply methods of improved perturbation theory ("boosted" coupling, "cactus" diagrams, etc.) to our results. Another direction is to compute $\mathscr{O}\left(a^{2} g^{2}\right)$ corrections to Green's functions; these, combined with non-perturbative evaluations, lead to an improvement in the non-perturbative estimates of the mixing coefficients.

Acknowledgments: The work of M. Constantinou and M. Costa was supported by the Cyprus Re-

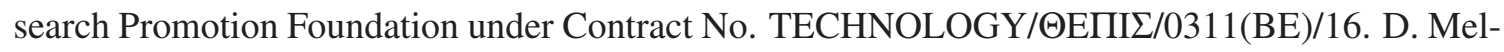
oni acknowledges MIUR (Italy) for financial support under the program Futuro in Ricerca 2010 (RBFR10O36O).

\section{References}

[1] M. Constantinou, M. Costa, R. Frezzotti, V. Lubicz, G. Martinelli, D. Meloni, H. Panagopoulos, S. Simula, A lattice study of the chromomagnetic operator, in preparation.

[2] G. D'Ambrosio, G. Isidori, G. Martinelli, PLB 480 (2000) 164 [arXiv: hep-ph/9911522].

[3] S. Bertolini, J.O. Eeg, M. Fabbrichesi, NPB 449 (1995) 197 [arXiv: hep-ph/ 9409437 ].

[4] D. Becirevic, V. Lubicz, G. Martinelli, F. Mescia, PLB 501 (2001) 98 [arXiv: hep-ph/ 0010349 ].

[5] I. Baum, V. Lubicz, G. Martinelli, L. Orifici, S. Simula, PRD 84 (2011) 074503 [arXiv:1108.1021].

[6] R. Frezzotti, G.C. Rossi, JHEP 0408 (2004) 007 [arXiv: hep-lat / 0306014 ]; JHEP 0410 (2004) 070 [arXiv: hep-lat/ 0407002 ]. 